\title{
Short-term wind power prediction based on time series analysis model
}

\author{
Wu Xiaodan ${ }^{1}$, Liu Wenying ${ }^{1}$ \\ ${ }^{1}$ Electrical \& electronic engineering school \\ North China electric power university \\ Beijing, China \\ wuxiaodan324@163.com
}

\author{
Wang Ningbo ${ }^{2}$, Ma Yanhong ${ }^{2}$ \\ ${ }^{2}$ Wind power technology center \\ Gansu electric power corporation \\ Lanzhou, Gansu, China \\ leehuadian@yeah.net
}

\begin{abstract}
In this paper, three kinds of prediction models based on time series analysis method are studied. Largely the process of building the wind farm short-term power forecasting model is discussed, mainly including sequence preprocessing, model identification, model order judging, parameter estimation, model testing and so on. It focuses on wind power short-term generation forecasting combined with the specific example using time series method. The example demonstrates the effectiveness and usefulness of the model.
\end{abstract}

Keywords-wind power; power forecast; time series method

\section{INTRODUCTION}

In recent years the wind power has always kept in high growth rate. But wind power has volatility, intermittency, low energy density and other characteristics, which make the wind farm output power volatile and intermittent. So studies must be conducted into generation power prediction of wind farm power to provide reliable bases for accepting wind power, reducing the peak-load adjustment cost, improving wind power quality and raising wind farms operation and maintenance efficiency.

Current prediction methods home and abroad include the physical prediction methods, statistical prediction methods and learning prediction methods, etc. Physical prediction methods based on digital weather forecasting have complex models and need large amount of calculations. So they are seldom used in short-term predictions. Statistics prediction methods are simple and require small amounts of data. So they are always used on occasions where data acquisition is relatively difficult. Artificial intelligence prediction methods are suitable for nonlinear stochastic system, but need to learn lots of individuals and require a large number of data.

Domestic wind power predictions at present mainly forecast wind speed and then the wind power which is converted from the speed. The output power of wind generator actually ranges in a wide area. It's not a curve but a bunch of curves. So this prediction method makes the predicting error of wind power bigger [1].

This paper proposes a time series analysis method from statistical forecasting methods to predict the short-term wind power directly, which reduces the error of wind speed conversion into wind power. The model is simple and requires small amounts of data.

The Key Research Program of China 863 Program (SQ2010AA0523193001).

\section{Introduction of TIME SERIES ANALYSIS}

Observation values $y\left(t_{1}\right), y\left(t_{2}\right), \cdots, y\left(t_{N}\right)$ of function $y(t)$ at $t_{1}<t_{2}<\cdots<t_{N}$ make up a discrete ordered set, which is called a time series recorded as $\{y(t)\}$. If the variable t means time, the group of observation data arranged in chronological order forms a time series. What needs to be pointed out is that the variable t does not always refer to time, and it can also be other variables whose value is monotonous increasing.

The time series is generated by a random process, namely a time sequence can be regarded as the actual reflection for a random process to be studied. Conversely, such a time sequence reflecting a random process also can be used to establish a proper mathematical model to describe the stochastic process. Establishing a suitable mathematical model from time series analysis is popularly discussed currently.

Time series prediction technology indicates that using a mathematical model to describe the sequence approximately which is formed from the forecasted object with the passage of time as a random sequence. Once this model is identified, we can predict the future values from the past and present values of the time series.

\section{TIme Series Analysis Forecasting ModeL}

According to Box-Jenkins method [2], stochastic time series prediction models can be classified as autoregressive models (AR), moving average models (MA) and autoregressive-moving average models (ARMA).

If the time sequence $\left\{y_{t}\right\}$ meets $y_{t}=\phi_{1} y_{t-1}+\cdots+\phi_{p} y_{t-p}+\varepsilon_{t}$, where the random variable sequence $\left\{\varepsilon_{t}\right\}$ is independently distributed and satisfies $E\left(\varepsilon_{t}\right)=0, \operatorname{Var}\left(\varepsilon_{t}\right)=\sigma^{2}>0$, then we can say the time series $\left\{y_{t}\right\}$ obeys the p order autoregressive model.

If the time sequence $\left\{y_{t}\right\}$ meets $\mathrm{y}_{t}=\varepsilon_{t}-\theta_{1} \varepsilon_{t-1}-\cdots-\theta_{q} \varepsilon_{t-q}$, then we can say the time series $\left\{y_{t}\right\}$ obeys the q order moving average model.

If the time sequence $\left\{y_{t}\right\}$ meets $y_{t}=\phi_{y_{t-1}}+\cdots+\phi_{p} y_{t-p}+\varepsilon_{t}-\theta_{1} \varepsilon_{t-1}-\cdots-\theta_{q} \varepsilon_{t-q}$, then we can say the time series $\left\{y_{t}\right\}$ obeys the (p, q) order autoregressive-moving average model.

To sum up, the AR model means that the present observation value is composed of a few past observation values and a current random disturbance; the MA model means that the present observation value is expressed by the linear combination of the random disturbances called white noise sequence. The ARMA model is made up of the AR model and the MA model. 


\section{Establish the SHORT-TiMe Wind POWER Time SERIES PREDICTION MODEL}

Because of the randomness and volatility of the wind, the wind power generation can be regarded as a random process. Wind power generations at different time can make up a chronological time series. Naturally it can be forecasted by the time series analysis. The short-term power prediction has a more obvious periodicity than medium and long-term predictions, so time series analysis is more suitable for the short-term wind farm power generation forecast. Discussion about the building of short-time wind power time series prediction model is as follows.

\section{A. Sequence Pretreatment}

First of all, we should get a group of historical observation values of short-term wind power generation, and regard it as the original time series for prediction. Because the time series is probably a non-stationary random sequence whose mean is not zero, we can't apply three time series prediction models to the series directly. Therefore, stationarity test is needed. If the sequence does not satisfy the stability requirements, differential smoothing will be required. The difference formula is: $W_{t}=\nabla^{d} Z_{t}$, where the corresponding $\mathrm{d}$ is the differential order number set to achieve smooth.

\section{B. Model Identification}

Model identification, that is choosing the suitable model from the AR, MA, ARMA to describe the stochastic wind power generation process that produces the practical sequence. And the characteristics of the random process can only be expressed by the original time series for prediction, so we must choose model according to the original time sequence. If the autocorrelation function of the original time series has "truncation" character, choose the MA model; If the partial correlation function of the original time series has "truncation" character, choose the AR model; If the autocorrelation function and partial correlation function both have "trailing" character, choose the ARMA model [3].

\section{Model Order Judging}

After the choice of a predictive model from the AR (p) model, MA (q) model and ARMA (p, q) model, we have to judge model order, that is, to determine the values of $\mathrm{p}$ and $\mathrm{q}$. The AIC method [4] can be used to get the values of $p$ and $q$ that make the AIC smallest. As a matter of fact, when the AIC is lowest, the residual variance of fitting function is least. At this time the model can give the original time series a optimal description, therefore, the present values of $\mathrm{p}$ and $\mathrm{q}$ are the best choice.

\section{Model Parameter Estimation}

After model identification and model order judging, the category, structure and order of the model have been determined primarily. The moment estimation method of parametric preliminary estimation and the least squares estimation method of parametric precision estimation can be used to estimate the unknown parameters of the model.

\section{E. Short-time Wind Power Time Series Prediction Model}

With the above steps, we can get the short-time wind power time series prediction model, and its form is one of the three models: $y_{t}=\phi_{1} y_{t-1}+\cdots+\phi_{p} y_{t-p}+\varepsilon_{t}$ (AR model), $\mathrm{y}_{t}=\varepsilon_{t}-\theta_{1} \varepsilon_{t-1}-\cdots-\theta_{q} \varepsilon_{t-q} \quad$ (MA model), $y_{t}=\phi_{1} y_{t-1}+\cdots+\phi_{p} y_{t-p}+\varepsilon_{t}-\theta_{1} \varepsilon_{t-1}-\cdots-\theta_{q} \varepsilon_{t-q}$ (ARMA model). Where, $y_{t}$ refers to the wind power to be predicted, $y_{t-1}, \cdots, y_{t-p}$ refer to the historical values of wind power, $\varepsilon_{t}, \varepsilon_{t-1}, \cdots, \varepsilon_{t-q}$ represent the random interferences of different moments, and $\phi_{1}, \cdots \phi_{p}, \theta_{1}, \cdots \theta_{q}$ are model parameters that they are obtained by parameter estimation. Model orders $\mathrm{p}$ and $\mathrm{q}$ are obtained by model order judging [3].

\section{F. Model Testing}

The residual sequence $a_{t}=x_{t}-x_{t}^{\prime}$ has to be checked after the determination of model order, where $x_{t}$ is the historical value of power generation, and $x_{t}^{\prime}$ is the model fitted value of power generation. If it is a sample sequence of white noise, then the model is appropriate, otherwise it is inappropriate. When it's improper, we need to modify or reproduce the forecasting model until the model passes the test [5].

After the above interactive steps, we can get a short-term wind power prediction model to predict the future values of wind power. Historical data need to be constantly updated as time goes by.

To make sure whether this model is appropriate rests with the actual prediction result of the wind power.

\section{EXAMPLE VERIFICATION}

This paper makes a detailed analysis of wind power generation time series of one wind farm in western china. The sequence is formed by sampling 1 point from each 10 minutes. Take the first 144 point data (i.e. wind power of a day) to set a model as the primitive data. The model will be used for short-term wind power prediction 10 minutes ahead.

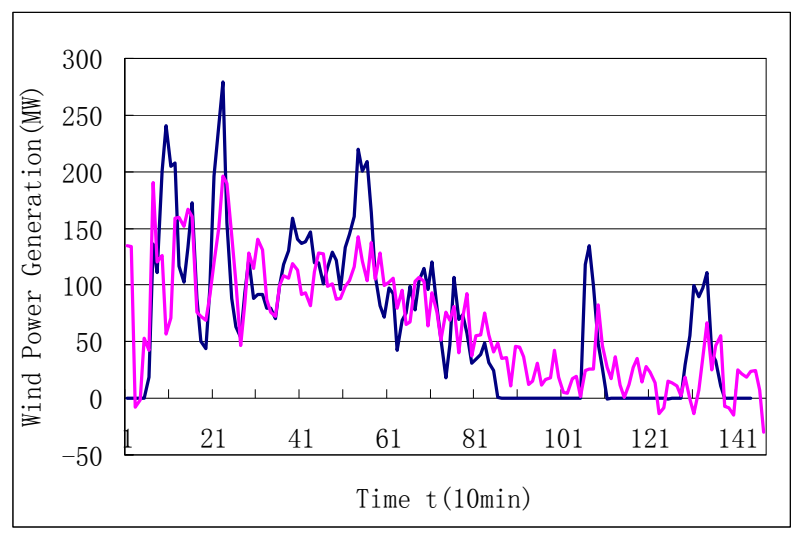

Figure 1. Predicted curves

Through the MATLAB programming, the model established by using the above method is ARMA $(1,4)$. Prediction results are shown in the above picture. The blue line represents the measured data, and the red line represents the model predicted data. Divide every six values of the predict data into a group (i.e. the values of every hour form a 
group), then we can get 24 groups. Absolute average error percentages between the predicted data and the measured data for each group are shown in the following table:

TABle I. Absolute Average Error Percentage

\begin{tabular}{|c|c|c|c|c|c|c|}
\hline $\begin{array}{c}\text { Group } \\
\text { Number }\end{array}$ & 1 & 2 & 3 & 4 & 5 & 6 \\
\hline Error & 0.0968 & 0.1879 & 0.1184 & -0.0713 & -0.0354 & -0.0303 \\
\hline $\begin{array}{c}\text { Group } \\
\text { Number }\end{array}$ & 7 & 8 & 9 & 10 & 11 & 12 \\
\hline Error & -0.0684 & 0.0838 & -0.0600 & -0.1628 & -0.2313 & -0.1522 \\
\hline $\begin{array}{c}\text { Group } \\
\text { Number }\end{array}$ & 13 & 14 & 15 & 16 & 17 & 18 \\
\hline Error & -0.0189 & 0.0667 & 0.0764 & 0.0416 & 0.1153 & -0.0090 \\
\hline $\begin{array}{c}\text { Group } \\
\text { Number }\end{array}$ & 19 & 20 & 21 & 22 & 23 & 24 \\
\hline Error & 0.0088 & -0.0244 & -0.0332 & -0.0179 & -0.0722 & 0.0642 \\
\hline
\end{tabular}

\section{CONCLUSION}

As can be seen from the example, the model using time series method to predict wind power is simple and requires a small amount of data. There are less errors and the model is suitable for situations where data acquisition has certain difficulty. But the prediction errors in some points of the prediction results are very large, especially in the time series turning points. That is because of the very strong randomness of wind speed and the difficulty to predict the occurrences of time turning point.
On the whole, this method has been proved to possess a high practical value with getting satisfactory results in most of the data points.

\section{ACKNOWLEDGMENT}

The authors sincerely thank the reviewers for their constructive comments and suggestions on improving the presentation.

\section{REFERENCES}

[1] Li Ran, Ke Yongqin, Zhang Xiaoqian and Tang Fan, "Wind power forecasting of wind farm based on time series and SVM," China Smart Grid Academic Seminar Symposium [C], 2011, pp.8-9.

[2] Wang Tongxu, Zhou Hongnian and Wang Yuan, “Applied BoxJenkins forecasting models and its application,” Journal of University of Electronic Science and Technology of China, vol.19, no.6, pp.635640, Dec 1990.

[3] Niu Dongxiao, Cao Shuhua, Zhao Lei and Zhang Wenwen, "Electric power load forecasting technology and its application,” China Electric Power Press, 1998, pp.97-119.

[4] Luo Qiaolin, "The AIC criterion about statistical recognition," Mathematics In Practice and Theory, 1981, pp.49-56.

[5] Gao Wenyuan, Liu Zunpeng, Wang Peng and Lu Xiaoguang, "Shortterm wind speed modeling based on time sequence method," Gansu Science and Technology, 2009, pp.14-16. 